\title{
Controle Temporal e Adaptabilidade Espacial na Resolução Numérica de uma Equação tipo KdV ${ }^{1}$
}

F.C.G. MENDONÇA2 2 Programa de Pós-Graduação em Computação Aplicada, Instituto Nacional de Pesquisas Espaciais - INPE, 12227-010 São José dos Campos, SP, Brasil.

M.O. DOMINGUES ${ }^{3}$, E.E.N. MACAU ${ }^{4}$, Laboratório Associado de Computação e Matemática Aplicada - LAC, Instituto Nacional de Pesquisas Espaciais - INPE, 12227-010 São José dos Campos, SP, Brasil.

Resumo. Nas últimas décadas, vários métodos vêm sendo desenvolvidos, utilizando ferramentas wavelet para resolução numérica de equações diferenciais parciais evolutivas com adaptabilidade espacial. Esses métodos, tradicionalmente, utilizam técnicas explícitas para a discretização no tempo. Com o aperfeiçoamento desses métodos espaciais, como por exemplo, os híbridos wavelets-diferenças finitas, há necessidade de enfoques explícitos temporais mais eficientes e estáveis. Com essa finalidade, são avaliados neste trabalho o uso de algumas técnicas de Runge-Kutta Encaixados (RKE) de ordem 4(5), nesse contexto adaptativo wavelet, para a resolução de um problema teste em uma equação tipo Korteweg-de Vries (KdV) com a interação de duas ondas solitárias.

Palavras-chave. Métodos numéricos, equações diferenciais, controle do passo de tempo, wavelet, equação de KdV.

\section{Introdução}

A técnica adaptativa espacial da Representação Esparsa de Pontos (SPR) [12] é um método geral para resolução de equações diferenciais parciais evolutivas (parabólicas ou hiperbólicas) que apresentem estruturas localizadas. A maioria desses problemas possui características que necessitam de uma adaptabilidade espacial mais elaborada para preservar a dinâmica do sistema modelado e manter a eficiência computacional. Por um lado, a utilização de uma malha pouco refinada fornece um resultado pouco preciso, devido à má representação da função próxima nas regiões com pouca regularidade, por outro lado, o custo computacional se torna inviável se todo domínio for refinado. Na simulação numérica ideal desse tipo de problema, é desejável que a discretização seja ajustável espacial e temporalmente e que haja

\footnotetext{
${ }^{1}$ Agradecimento por auxílio FAPESP.

${ }^{2}$ flaviacgm@gmail.com

${ }^{3}$ margarete@lac.inpe.br,margarete.oliveira.domingues@gmail.com.

${ }^{4}$ elbert@lac.inpe.br.
} 
um controle do erro na evolução da solução. Por esses motivos, diferentes métodos têm sido desenvolvidos na tentativa de associar a adaptabilidade do passo de tempo com a adaptabilidade da discretização espacial [3, 5]. Por exemplo em [6, 5, 4, 9] é proposto um refinamento de malhas adaptativo acoplado com um método de RungeKutta encaixado explícito para escolher automaticamente o tamanho do passo de tempo enquanto controla o erro.

Para avaliar o desempenho computacional da SPR com alguns dos métodos de controle temporal Runge-Kutta encaixados (RKE) mais populares utilizou-se neste trabalho uma equação tipo Korteweg-de Vries $(K d V)$ com duas ondas solitárias. Nessas equações não-lineares ocorre variação na regularidade da solução durante a evolução temporal em diferentes regiões do domínio a medida que essas ondas se encontram e se propagam novamente sem perda de suas formas originais. Sendo assim, elas são bons exemplos de estudo da adaptabilidade espacial dinâmica da malha à solução, uma das principais características de aplicação aos métodos SPR.

É conhecido também que o passo de tempo deve ser muito pequeno nesses tipos de equação, o que introduz erros numéricos na própria evolução temporal da solução. Essas características das KDVs as tornam equações de interesse para estudo simultâneo do comportamento do método de adaptabilidade espacial em que o controle temporal pode melhorar o desempenho da respresentação da solução. Em especial, esse problema teste tem uma solução analítica o que possibilita uma avaliação do erro cometido nas discretizações e nos diversos métodos discutidos.

Nas próximas seções, uma breve introdução ao método SPR e aos métodos RKE é apresentada. A seguir, a Equação de KdV é descrita, bem como os resultados obtidos na resolução da mesma, utilizando esse método adaptativo espacial em conjunto com os métodos de controle do passo de tempo, visando à utilização de técnicas mais eficientes computacionalmente e com aplicações a outros esquemas de interesse.

\section{Objetivo}

Este trabalho é dedicado à avaliação do desempenho numérico e computacional de esquemas adaptativos temporais explícitos de ordem 4(5), juntamente com esquemas wavelet adaptativos espaciais, utilizando o método de Representação Esparsa de Pontos em soluções com diferentes regularidades localizadas no domínio e que são alteradas durante a evolução temporal.

\section{Método de Representação Esparsa de Pontos}

Nas aplicações deste trabalho, considera-se a SPR, técnica proposta por [12] e utilizada em [3, 14]. Nela, um esquema tradicional de diferenças finitas em uma malha uniforme é associado à discretização com adaptação de funções por valores pontuais. Os pontos restantes, na malha adaptativa, estão associados aos coeficientes wavelet menores que um certo valor de truncamento. Nessa técnica, apesar de não estarem todos os valores pontuais presentes nas malhas, eles podem ser eficientemente recuperados dada a representação de valores existente. Nesse contexto, a 
análise wavelet interpolatória contribui tanto na construção de tais malhas, quanto na construção de um esquema de interpolação adaptável para fazer a conexão entre os contextos uniforme e o não-uniforme. O principal ingrediente consiste de um algoritmo de refinamento interpolante que, a partir das informações correspondentes a um certo nível, fornece aproximações para o nível seguinte mais refinado, interpolando os pontos antigos. Os coeficientes wavelet são as diferenças de informação entre os valores exatos e os valores aproximados, fornecidos pelo algoritmo de refinamento nos pontos novos, ou seja, erros de interpolação. O princípio básico é que os coeficientes wavelet podem ser utilizados como indicadores de regularidade local das funções, sendo pequenos em regiões de suavidade e significativos em regiões de variação brusca. Supondo que, em um determinado tempo, seja dada uma representação da solução em uma malha adaptativa, com a variação temporal da posição dessas estruturas, faz-se necessário que seja possível redefinir as malhas, dinâmica e automaticamente, durante as simulações numéricas, assim como ajustar o passo de tempo da forma mais eficiente possível às novas malhas, de modo a se obter melhores resultados no desempenho do método.

No estudo realizado, vários parâmetros de truncamento foram utilizados, sendo que neste trabalho para facilitar as comparações apenas os resultados obtidos com o parâmetro $\epsilon=10^{-4}$ são apresentados. A escolha de tal parâmetro se deve a um balanço entre a precisão requerida e a quantidade de pontos da malha adaptativa, o que foi determinado após a realização de experimentos numéricos, cuja descrição extrapola o contexto deste artigo.

\section{Métodos de Runge-Kutta Encaixados}

Na tentativa de se implementar procedimentos que permitam ajustar adaptativamente o passo de integração em um método RK, foram criadas as Fórmulas RKE [15]. Sejam as soluções $y_{1}, \hat{y}_{1}$ de ordem $p$ e $p+1$, respectivamente expressas por

$$
y_{1}=y_{0}+\triangle t\left(b_{1} k_{1}+\ldots+b_{s} k_{s}\right), \quad \hat{y}_{1}=y_{0}+\Delta t\left(\hat{b}_{1} k_{1}+\ldots+\hat{b}_{s} k_{s}\right),
$$

em que $b$ e $\hat{b}$ são coeficientes e $k_{s}$ é função dos coeficientes $c_{i}$. O algoritmo, que ajusta automaticamente o tamanho do passo, tem como objetivo determinar um passo de integração que satisfaça a tolerância prescrita do erro local [11], visando alcançar a precisão pré-determinada, na solução, com o mínimo esforço computacional, ou seja, com o maior passo possível. Quando um tamanho de passo inicial é escolhido, o algoritmo calcula duas aproximações para a solução, $y_{1}$ e $\hat{y}_{1}$. Então, uma estimativa do erro resulta de $\left|y_{1}-\hat{y}_{1}\right|$. É esta diferença que deve ser mantida a um grau de precisão desejado. Isso é feito ajustando-se o tamanho de $\Delta t$. O estimador do erro pode então ser escrito como $\Delta=y_{1 i}-\hat{y}_{1 i}=\sum_{i=1}^{\hat{p}}\left(b_{i}-\hat{b}_{i}\right) k_{i}$. Dado um passo inicial $\Delta t_{\text {inicial }}$, denota-se o erro por ele produzido por $\Delta_{\text {inicial }}$. Do mesmo modo, um novo passo $\Delta t_{\text {novo }}$ produzirá um erro $\Delta_{\text {desejado. Ambos estão relacionados por }}$ $\frac{\Delta t_{\text {novo }}}{\Delta t_{\text {inicial }}}=\left|\frac{\Delta_{\text {desejado }}}{\Delta_{\text {inicial }}}\right|^{\alpha}$, em que $\Delta_{\text {desejado denota a precisão desejada e } \alpha \text { pode }}$ assumir valores como $\left(\alpha_{0}, \alpha_{1}\right)=\left(\frac{1}{p}, \frac{1}{p+1}\right)[4,15]$. Se $\Delta_{\text {inicial }}<\Delta_{\text {desejado, tem-se }}$ o quanto se pode aumentar o passo de tempo de forma segura no próximo passo. 
Neste caso, $\alpha=\alpha_{1}$ é escolhido. Por outro lado, se $\Delta_{\text {inicial }} \geq \Delta_{\text {desejado, tem-se o }}$ quanto se deve diminuir o tamanho do passo de tempo para uma nova tentativa, sendo $\alpha=\alpha_{0}$ escolhido.

Esse procedimento permite ajustar automaticamente o tamanho do passo, de modo a encontrar uma precisão prescrita no tempo. Contudo, quando $\Delta t_{\text {novo }}$ sofre um incremento muito grande, o novo valor predito pode falhar para encontrar a precisão desejada. Dessa forma, uma medida para evitar esse tipo de problema é limitar o aumento do passo de tempo, introduzindo um fator de segurança $S$. Com isso, aumenta-se a probabilidade de aceite do próximo passo de tempo, evitando o aumento ou a diminuição demasiada do tamanho de $\Delta t$, e.g., valores freqüentemente adotados para $S$ são 0.85 e 0.9 , i.e., uma redução de 10 a $15 \%$ da estimativa de $\triangle t$ [11].

\subsection{Métodos Implementados}

Três métodos RKE explícitos de ordem 4(5), utilizados tradicionalmente na resolução de Equações Diferencais Ordinárias (EDOs), são analisados neste trabalho: os métodos de Runge-Kutta Fehlberg (F45), Cash $\&$ Karp (CK45) e Dormand e Prince (DP45). De modo tradicional, os coeficientes de Runge-Kutta são apresentados em tabelas, veja [11]. Como exemplo, os coeficientes dos métodos F45 e CK45 estão apresentados nas Tabelas 1 e 2 .

As simulações são realizadas com 513 pontos e uma tolerância $\Delta_{\text {desejado }}=10^{-4}$ nos métodos RKE 4(5).

Tabela 1: Coeficientes para Fehlberg 4(5)

\begin{tabular}{c|cccccc}
0 & & & & & & \\
$1 / 4$ & $1 / 4$ & & & & & \\
$3 / 8$ & $3 / 32$ & $9 / 32$ & & & & \\
$12 / 13$ & $1932 / 2197$ & $-7200 / 2197$ & $7296 / 2197$ & & & \\
1 & $439 / 216$ & -8 & $3680 / 513$ & $-845 / 4104$ & & \\
$1 / 2$ & $-8 / 27$ & 2 & $-3544 / 2565$ & $1859 / 4104$ & $-11 / 40$ & \\
\hline & $25 / 216$ & 0 & $1408 / 2565$ & $2197 / 4104$ & $-1 / 5$ & 0 \\
\hline & $16 / 135$ & 0 & $6656 / 12825$ & $28561 / 56430$ & $-9 / 50$ & $2 / 55$
\end{tabular}


Tabela 2: Coeficientes para Cash \& Karp 4(5)

\begin{tabular}{c|cccccc}
0 & & & & & & \\
$1 / 5$ & $1 / 5$ & & & & & \\
$3 / 10$ & $3 / 10$ & $9 / 40$ & & & & \\
$3 / 5$ & $3 / 10$ & $-9 / 10$ & $6 / 5$ & & & \\
1 & $-11 / 54$ & $5 / 2$ & $-70 / 27$ & $35 / 27$ & & \\
$7 / 8$ & $1631 / 55296$ & $175 / 512$ & $575 / 13824$ & $44275 / 110592$ & $253 / 4096$ & \\
\hline & $37 / 378$ & 0 & $250 / 621$ & $125 / 621$ & 0 & $512 / 1771$ \\
\hline & $2825 / 27648$ & 0 & $18575 / 48384$ & $13525 / 55296$ & $277 / 14336$ & $1 / 4$
\end{tabular}

\section{Equação de Korteweg-de Vries}

A equação de Korteweg-de Vries (KdV) de interesse é expressa como

$$
\frac{\partial \mathcal{U}}{\partial t}-6 \mathcal{U} \frac{\partial \mathcal{U}}{\partial x}+\frac{\partial^{3} \mathcal{U}}{\partial x^{3}}=0
$$

em que $\mathcal{U}=\mathcal{U}(x, t)$, para $t \geq 0, x \in[-20,20]$. O exemplo considerado é apresentado em [17]. Nele as condições de fronteira são periódicas e a condição inicial é dada por $\mathcal{U}(x, 0)=-6 \operatorname{sech}^{2}(x)$. A solução analítica (exata) apresenta duas ondas solitárias ${ }^{5}$ deslocando-se pelo domínio e tem a seguinte expressão

$$
\mathcal{U}(x, t)=-12 \frac{3+4 \cosh (2 x-8 t)+\cosh (4 x-64 t)}{[3 \cosh (x-28 t)+\cosh (3 x-36 t)]^{2}} .
$$

A Figura 1 apresenta a evolução temporal da solução numérica para os tempos $t=0$ e $t=0.4$, respectivamente. Em a), a solução é dada para o tempo $t=0$, momento em que os duas ondas solitárias se encontram. Em b), as duas ondas se afastam presevando suas formas originais. Nessa solução numérica, esquemas de diferenças finitas centradas de ordem 4 são utilizados com a condição de estabilidade (condição tipo de CFL) de $\frac{\Delta t}{(\Delta x)^{3}}<\frac{1}{\pi^{3}} \approx 0.03225$, conforme as estimativas apresentadas em [10].

\footnotetext{
${ }^{5}$ Os solitons são ondas solitárias que mantêm sua forma à medida que o tempo passa.
} 
(a) $t=0$

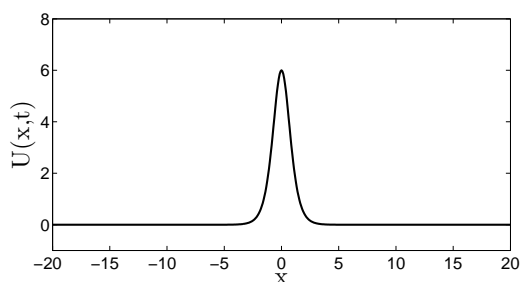

(b) $t=0.4$

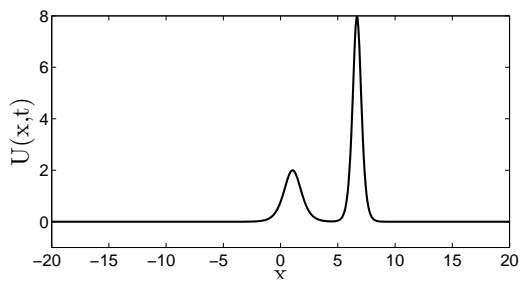

Figura 1: Solução numérica da Equação de KdV estudada para (a) $t=0$ e (b) $t=0.4$.

\section{Resultados}

A Figura 2 corresponde às malhas adaptativas obtidas pelos métodos CK45 e F45, ilustrando a posição dos coeficientes "wavelet"' de valor significativo. O eixo $x$ corresponde ao domínio considerado na simulação e o eixo $y$ apresenta os diferentes níveis de refinamento utilizados para representar o problema em questão.

(a) CK45

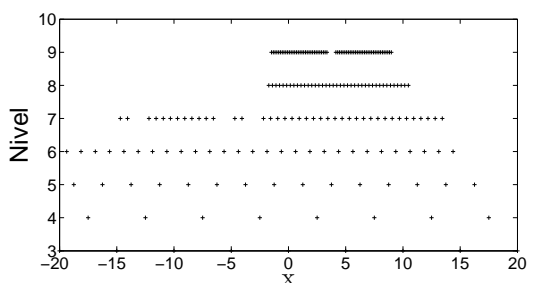

(b) F45

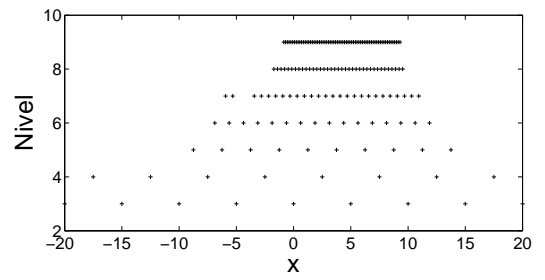

Figura 2: Malhas adaptativas obtidas na resolução da Equação de KdV utilizando os métodos RKE 4(5) em $t=0.4, \operatorname{com} \epsilon=10^{-4}$ e $\Delta_{\text {desejado }}=10^{-4}$ : (a) Método CK45 e (b) Método F45.

Na Figura 1, em b), é apresentada a solução numérica da equação para o tempo $t=0.4$. Nesse momento, a solução apresenta duas regiões onde encontram-se as ondas solitárias, aproximadamente no intervalo $[0,10]$ do domínio utilizado. Dessa forma, as malhas adaptativas, ilustradas na Figura 2, são refinadas nesta região. A Figura 3 ilustra o número de coeficientes "'wavelet"' de valor significativo $\left(N_{s}\right)$ utilizados por cada um desses métodos RKE 4(5) durante a evolução do modelo até o tempo $t=0.4$.

Observa-se, na Figura 3, que, para resolução da Equação de KdV, esses métodos RKE de ordem 4(5) utilizam, a princípio, aproximadamente 120 pontos. Durante a evolução, o método CK45 chega a atingir um valor máximo de 250 pontos. Já os métodos F45 e DP45 utilizam, durante a evolução temporal, o valor máximo de 190 pontos ao longo de todo o processo de integração. Esses resultados evidenciam o ganho obtido com o uso de métodos adaptativos, uma vez que, o número máximo de pontos necessários nestes métodos não chega nem a ser a metade dos pontos 


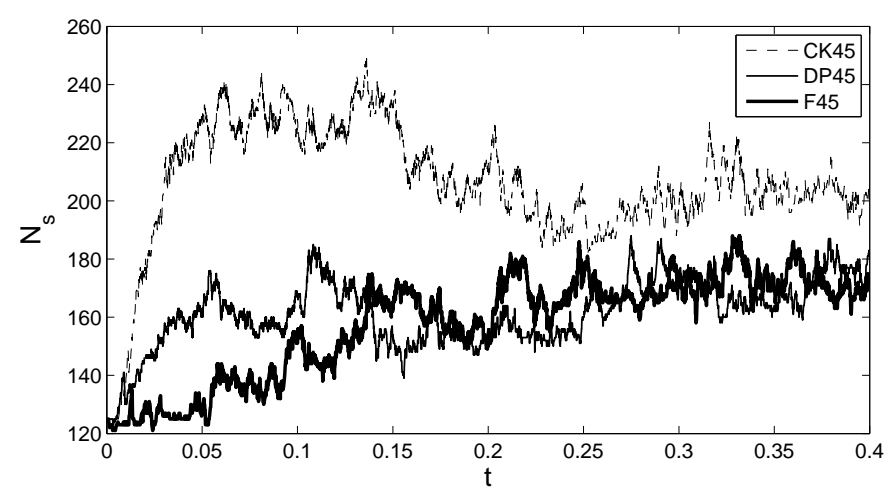

Figura 3: Número de pontos significativos $\left(N_{s}\right)$ presentes na malha adaptativa durante a evolução temporal da Equação de KdV utilizando os três métodos RKE 4(5) estudados.

utilizados ao se recorrer a métodos não adaptativos espacialmente.

Neste contexto numérico, estamos interessados na análise comparativa dos três métodos RKE 4(5) considerados. A Tabela 3 apresenta os resultados obtidos com a utilização de métodos tradicionais, no espaço (DF) e no tempo (RK4), e do método RK4, associado à SPR, até $t=0.4$. Nesses métodos, o passo de tempo utilizado corresponde ao menor passo de tempo encontrado pelos métodos RKE 4(5), ou seja, $\triangle t_{\text {min }}=1.4305 \cdot 10^{-5}$.

Na Tabela 3, pode-se observar que ambos os métodos realizam o mesmo número de passos, apresentando, assim, a mesma complexidade. Os métodos tradicionais são mais precisos na resolução do problema. Essa precisão vem acompanhada de um tempo de processamento três vezes superior ao tempo do método SPR. A percentagem do tempo de CPU apresentada corresponde ao tempo de CPU gasto por RK4 e DF, que são métodos tradicionais na resolução de EDPs.

Tabela 3: Desempenho dos métodos tradicionais e do método RK4 associado à SPR na solução da Equação de KdV. Parâmetros considerados: número de passos realizados, erro cometido $L_{\infty}$, complexidade computacional e tempo de CPU .

\begin{tabular}{|c|c|c|c|c|c|}
\hline Método & Passos & $L_{\infty}$ & Complexidade & \multicolumn{2}{|c|}{ CPU } \\
\hline & & & & seg & $(\%)$ \\
\hline \hline RK4 e DF & 27963 & 0.0010 & 195741 & 4586.13 & 100.0 \\
\hline RK4 e SPR & 27963 & 0.0437 & 195741 & 1470.78 & 32.07 \\
\hline
\end{tabular}

Os métodos de controle do passo de tempo CK45, DP45 e F45 têm seus desempenhos apresentados na Tabela 4. A fim de se obter melhores resultados no desempenho de cada método, foram feitos testes variando o valor do fator de segurança $S$ e do parâmetro $\alpha$. As percentagens da complexidade e do tempo de CPU apresentadas correspondem aos valores obtidos pela SPR e RK4, apresentados na 
Tabela 3, utilizando o menor passo de tempo encontrado pelos métodos RKE 4(5). Pode-se observar que todos os métodos apresentam um número maior de passos bons do que de passos ruins. Portanto, a previsão dos passos de tempo é adequada na maior parte do tempo no processo de integração. Ocorre uma redução do número de passos ruins quando $S=0.85$. Dentre os três métodos RKE, o método F45 se destaca em quase todos os casos com o menor erro cometido. Os métodos RKE se mostram menos custosos do que o método RK4 com e sem adaptabilidade espacial. Em todos os casos, o método DP45 se apresenta como o método mais custoso dentre os métodos RKE 4(5). O método F45 se apresenta como o método de menor custo.

Tabela 4: Desempenho dos métodos RKE 4(5) na solução da Equação de KdV.

\begin{tabular}{|c|c|c|c|c|c|c|c|c|c|}
\hline Método & \multicolumn{2}{|c|}{ Passos } & $S$ & $\alpha$ & $L_{\infty}$ & \multicolumn{2}{|c|}{ Complexidade } & \multicolumn{2}{|c|}{ CPU } \\
\hline & Bons & Ruins & & & & valor & $(\%)$ & seg & $(\%)$ \\
\hline \hline CK45 & 4022 & 793 & 0.9 & -0.2 & 0.0215 & 33705 & 17.2 & 474.4 & 32.25 \\
\hline DP45 & 4211 & 808 & 0.9 & -0.2 & 0.0175 & 40152 & 20.5 & 457.01 & 31.07 \\
\hline F45 & 4488 & 612 & 0.9 & -0.2 & 0.0114 & 30600 & 15.6 & 353.3 & 24.02 \\
\hline \hline CK45 & 5201 & 89 & 0.85 & -0.1 & 0.0148 & 37030 & 18.9 & 363.0 & 24.68 \\
\hline DP45 & 5840 & 103 & 0.85 & -0.1 & 0.0114 & 47544 & 24.3 & 457.71 & 31.12 \\
\hline F45 & 5815 & 36 & 0.85 & -0.1 & 0.0159 & 35106 & 17.9 & 373.29 & 25.38 \\
\hline \hline CK45 & 4026 & 402 & 0.85 & -0.2 & 0.0189 & 30996 & 15.8 & 339.85 & 23.11 \\
\hline DP45 & 5477 & 401 & 0.85 & -0.2 & 0.0217 & 47024 & 24.0 & 506.08 & 34.41 \\
\hline F45 & 4996 & 491 & 0.85 & -0.2 & 0.0116 & 32922 & 16.8 & 373.71 & 25.41 \\
\hline \hline CK45 & 5231 & 777 & 0.95 & -0.1 & 0.0221 & 42056 & 21.5 & 633.66 & 43.08 \\
\hline DP45 & 5865 & 568 & 0.95 & -0.1 & 0.0218 & 51464 & 26.3 & 692.56 & 47.09 \\
\hline F45 & 4362 & 464 & 0.95 & -0.1 & 0.0147 & 28956 & 14.8 & 319.86 & 21.75 \\
\hline \hline CK45 & 3546 & 1458 & 0.95 & -0.2 & 0.0281 & 35028 & 17.9 & 586.29 & 39.86 \\
\hline DP45 & 4799 & 1470 & 0.95 & -0.2 & 0.0158 & 50152 & 25.6 & 698.47 & 47.49 \\
\hline F45 & 3367 & 1431 & 0.95 & -0.2 & 0.0125 & 28788 & 14.7 & 366.14 & 24.89 \\
\hline
\end{tabular}

\section{Considerações Finais}

Grosso modo, um método ideal seria aquele que minimizasse erros numéricos, enquanto nunca gerasse passos considerados ruins. No entanto, essas características não se encontram unificadas em um só método.

Nos casos estudados, constatou-se que a utilização do método tradicional de DF associado ao RK4 forneceu uma solução mais próxima da solução analítica (exata) do problema. No entanto, o ganho em precisão vem acompanhado de um custo e um tempo de processamento cerca de 3 a 4 vezes maior. Por outro lado, os métodos RKE associados à SPR são estratégias mais precisas que o método RK4 e a SPR, o que os torna técnicas eficientes na resolução numérica de um determinado problema, ao fornecer uma maior eficiência computacional.

De maneira geral, os métodos de ordem 4(5) obtiveram erros relativamente próximos, sendo todos eles inferiores ao erro obtido por RK4 associado à SPR. 
Dentre os métodos de ordem 4(5), o método F45 se apresentou como o método menos custoso obtendo ainda os menores erros na maior parte dos casos.

Diante disso, o método F45 é selecionado como o método mais adequado dentre os estudados para esse contexto adaptativo, pois apresenta o melhor balanço na variação entre o número de passos, o erro e a complexidade computacional. Isto é, ele teve uma performace mais constante do que os outros métodos estudados, apresentando um bom desempenho em todas as análises. Conclui-se, assim, que a utilização de métodos adaptativos é uma estratégia eficiente tanto do ponto de vista computacional quanto numérico.

\title{
Agradecimentos
}

Os autores agradecem à FAPESP (proc.: 2005/03615-0) a bolsa de Mestrado de F. C. G. de Mendonça na CAP/INPE; à Universidade de Bio-Bio e à FAPESP o apoio financeiro a visita técnica de F. C. G. Mendonça à Universidade de Bio-Bio e Universidade de Concepcion, no Chile, onde discussões sobre essa aplicação foram realizadas; ao PROAP-CAPES/CAP-INPE, o apoio a participação no DINCON 2008; e à Dra. Sônia Maria Gomes, ao Dr. Octavio Vera e ao Dr. Mauricio Sepúlveda, as discussões científicas na parte teórica e de aplicação. E. E. N. Macau e M. O. Domingues agradecem ao CNPq pelo suporte as suas pesquisas.

\begin{abstract}
Wavelet methodology has been exploited as the key tool embedded on the up-to-date methods that are specially tailored to numerically solve with spatial adaptability evolved partially differential equations. Traditionally, these methods use explicit procedures to deal with time discretization. However, the enhancement of this approach requires the development of more efficient and stable explicit temporal techniques. In this work, we assess the performance that can be draw by using some Runge-Kutta embedded (RKE) techniques of 4(5) order in this scenario of adaptive wavelet to numerically solve the Korteweg-de Vries (KDV) equation.
\end{abstract}

\section{Referências}

[1] K. Baumgärtel, Soliton approach to magnetic holes, Journal of Geophysical Research, 104, No. A12 (1999), 28295-28308.

[2] D.R. Christie, K.J. Muirhead, A.L. Hales, On solitary waves in the atmosphere, Journal of the Atmospheric Sciences, 35, No. 5 (1978), 805-825.

[3] M.O. Domingues, "Análise Wavelet na Simulação Numérica de Equações Diferenciais Parciais com Adaptabilidade Espacial", UNICAMP, 2001.

[4] M.O. Domingues, O. Roussel, K. Schneider, On space-time adaptive schemes for the numerical solution of PDEs, ESIAM Proceedings, 16 (2007), 181-194.

[5] M.O. Domingues, S.M. Gomes, O. Roussel, K. Schneider. An adaptive multiresolution scheme with local time stepping 3 for evolutionary PDEs, Journal of Computational Physics, 227 (2008), 3758-3780. 
[6] M. O. Domingues, O. Roussel, K. Schneider. An adaptive multiresolution method for parabolic PDEs with time-step control. International Journal for Numerical Methods in Engineering, 2008 (em publicação).

[7] M. O. Domingues, S. M. Gomes, O. Roussel, K. Schneider. Space-time adaptive multiresolution methods for hyperbolic conservation laws: Applications to compressible Euler equations. Applied Numerical Mathematics, 2008(aceito).

[8] E. Fehlberg, "Klassische Runge-Kutta Formeln Fünfter und Siebenter Ordnung mit Schrittweiten-Kontrolle", Computing., 4, p.93-106, 1969.

[9] L. Ferm, P. Löstedt, Space-time adaptive solutions of first order PDEs, Journal on Scientific Computing, 26, n.1, p.83-110, 2006.

[10] B. Fornberg, A practical guide to pseudoespectral methods, Cambridge University Press, 1996.

[11] E. Hairer, S. P. Norsett, G. Wanner, "Solving Ordinary Differential Equations I - Nonstiff Problems", Springer-Verlag Berlin Heidelberg, Second Revised Edition, 1993.

[12] M. Holmström, Solving hyperbolic PDEs using interpolating wavelets, SIAM Journal on Scientific Computing, 21, p.405-420, n.2, 1999.

[13] A. Nunes, M. T. Gama, "Prisma - À Luz da Física", Centro de Física Teórica e Computacional, 2007.

[14] P. Pinho, M. O. Domingues, P. J. S. G. Ferreira, S. M. Gomes, A. Gomide, J. R. Pereira, Interpolating wavelets and adaptive finite difference schemes for solving maxwell's equations: gridding effects, IEEE Transactions on Magnetics, 43, p.1-10, 2007.

[15] W. H. Press, S. A. Teukolsky, W. T. Vetterling, B. P. Flannery, "Numerical Recipes in $\mathrm{C}++$ - The Art o Scientific Computing", Cambridge University Press, Second Edition, 2002.

[16] A. Rees, A. Balogh, T.S. Horbury, Small-scale solitary wave pulses observed by ulisses magnetic field experiment, Journal of Geophysical Research, 11, A10106, doi:10.1029/2005JA011555, 2006.

[17] J. Y. Yang, C. J. Chiou, Wavelet solution of the Korteweg-de Vries equation, Institute of Applied Mechanics - National Taiwan University, p.44. 